\title{
A multi-view image rectification algorithm for matrix camera arrangement
}

\author{
Jiachen Yang*1,2, Fei Guo ${ }^{1}$, Huogen Wang ${ }^{1}$, Zhiyong Ding ${ }^{1}$ \\ ${ }^{1}$ School of Electronic Engineering, Tianjin University, Tianjin, China \\ ${ }^{2}$ Tianjin Yaan Polytron Technologies Inc, Tianjin, China
}

Received: September 25, 2013

DOI: $10.5430 /$ air.v3n1p18
Accepted: October 28, $2013 \quad$ Online Published: November 21, 2013

URL: http://dx.doi.org/10.5430/air.v3n1p18

\begin{abstract}
This paper presents a rectification algorithm for multi-view images captured by a matrix multi-camera arrangement. In this work, an ergodic method is proposed to trace the ideal rotation matrix and the preferred location of the optical centers. Moreover, the camera's intrinsic parameters are optimized to obtain the ideal projection matrix, from which the image rectifying transform matrix(IRTM) is further derived. Then the multi-view image is rectified with the IRTM. Experimental results show that this proposed algorithm has great effect on multi-view image rectification for matrix camera arrangement.
\end{abstract}

Key Words: Image rectification, Multi-view images, Matrix multi-camera arrangement, Ergodic method

\section{Introduction}

With the rapid development of free video TV (FTV) and 3DTV, multi-view video technology has attracted a wide attention. ${ }^{[1-3]}$ ISO/IECJTC1/SC29/WG11 moving picture experts group (MPEG) has carried out in-depth investigations in the aspects of generation and representation of the multiview video, and has set some related standards. ${ }^{[4-6]}$ By providing the viewer with a plurality of viewpoints, multi-view video enables us to view 3D space by freely changing our viewpoints, ${ }^{[1]}$ and to experience three-dimensional and immersive feelings. ${ }^{[7-9]}$ Recently, the common way to obtain the multi-view video for a real-time FTV system is onedimensional capturing system which has a simple structure, such as parallel camera arrangement in which all of cameras are collinear. ${ }^{[10,11]}$ Compared with parallel camera arrangement, matrix camera arrangement is a two-dimensional capturing system in which cameras in row and cameras in column are collinear and even-distributed respectively. ${ }^{[12]}$ The images captured by matrix camera arrangement have not only horizontal parallax but also vertical parallax, which will be helpful to provide more naturally three-dimensional and immersive feeling. Besides, the multi-video captured by matrix camera arrangement can support multiple viewers to experience 3-D sensation consistent with their respective position in front of the display. Moreover, based on this configuration and existing techniques from the image mosaicing literature, we can register and blend the images captured by matrix camera arrangement to create a single image of high resolution. And each camera adjusts its exposure independently, allowing us to record high dynamic range environments. ${ }^{[13]}$ However, due to the stent deformation, different intrinsic characteristics of cameras and manual adjustment errors, some image defects, such as the vertical parallax and the inequality of horizontal disparity between adjacent pairs of images in row, and the horizontal parallax and the inequality of vertical disparity between adjacent pairs of images in column for multi-view images captured by matrix camera arrangement, are inevitably produced. These deficiencies seriously influenc the quality of stereo images produced by the back-end system. ${ }^{[14]}$ Therefore, the multi-view image rectification is of great significance in the production of multi-view video.

In the field of computer vision undergoing rapid development, many image rectification methods were proposed. However, most of them are binocular and trinocular rectification methods, ${ }^{[15-19]}$ such as binocular rectification method

*Correspondence: Dr. Jiachen Yang; Email: yangjiachen@tju.edu.cn; Address: School of Electronic Engineering, Tianjin University, Tianjin, ON., 300072, China. 
based on polar or homography ${ }^{[15-17]}$ and trinocular rectification method by projection invariance. ${ }^{[20,21]}$ In recent years, multi-view image rectification is becoming a research focus, and it undergoes a rapid development especially in the aspect of multi-view image rectification based on camera calibration. Ekmekcioglu et al. directly extended binocular rectification algorithm to multi-view situation. Taking the image captured by the middle camera of the parallel camera array as reference image, they used binocular rectification method to rectify the images captured by other cameras, and finally obtained the rectified Multi-view images. ${ }^{[6]}$ Kim et al. calculated an ideal camera arrangement using the minimization of global re-projection error between inter-view corresponding points. Then they obtained the rectified images by applying the homography matrix. ${ }^{[22]}$ Boutarel et al. obtained the horizontal axis by the least square method of all the camera centers and averaged the original optical axes to generate the common optical axes. ${ }^{[23]}$ Ho et al. repeatedly connected each midpoint from the original camera centers to calculate the line as the common horizontal axis for rectified multi-view images. ${ }^{[24]}$ Ho et al. improved the algorithm based on their fundamental work. Using iterative calculation, they adopted a camera's rotation matrix of the camera array as the ideal rotation matrix, and selected a camera's optical center as the reference to calculate the ideal optical centers of other cameras, then they calculated the homography matrix to rectify multi-view images. ${ }^{[25]} \mathrm{In}$ previous algorithms for parallel camera arrangement, there is an opportunity that the output images have visual distortion such as image slant. These images are rectified based on the line which either minimizes the sum of absolute difference from each camera centers or is confirmed by a camera's horizontal axis, so that the unexpected basic axes can occur in the rectified multi-view image geometry. In this case, although geometric errors are reduced, the visual quality of the rectified multi-view image captured by parallel camera arrangement is very low as auto-stereoscopic input.

At present, most of the multi-view image rectification methods are applied to parallel multi-camera array. These methods are mainly to calculate the common horizontal axis, and ensure that all ideal optical centers locate to it and the distance between adjacent pairs of cameras is equivalent. Moreover, the optical axes of all cameras are paralleled and perpendicular to the common horizontal axis. The image rectification method for matrix camera arrangement should guarantee that the cameras in row and cameras in column are collinear and even-distributed respectively. Moreover, all of the optical axes should be paralleled and perpendicular to the common horizontal axis and vertical axis. As far as I know, there is no image rectification algorithm for matrix camera arrangement. In this paper, we use an ergodic method to find out the ideal rotation matrix and the ideal location of every optical center, and get the ideal projective matrix that makes the matrix camera arrangement satisfy the ideal condition. Then we calculate the image rectifying transform matrix (IRTM) by the homography between the original and ideal matrix camera arrangement. By applying the IRTM to the original multi-view images captured by matrix camera arrangement, we obtain the rectified images. Experimental results show that our algorithm availably eliminates the vertical parallax and the inequality of horizontal disparity between adjacent pairs of images in row, meanwhile, effectively eliminates the horizontal parallax and the inequality of vertical disparity between adjacent pairs of images in column.

The paper is organized as follows. In Section 2, we formulate the multi-view image rectification algorithm. And in Section 3, we lay out the experiment details and perform the data analysis. Finally, in Section 4, we reach a conclusion.

\section{Multi-view image rectification algorithm}

In this section, we proposed an image rectification algorithm for the matrix multi-camera arrangement. This algorithm aims to decrease the geometric errors and the influence caused by different intrinsic characteristics of different cameras, and finally gets the rectified multi-view images, which achieve the quality of the images captured by the ideal matrix multi-camera arrangement. We firstly introduce the matrix camera arrangement briefly, and then present our multi-view image rectification algorithm.

\subsection{Introduction of matrix multi-camera arrange- ment}

Figure 1 shows a matrix multi-camera arrangement projective model, each camera is located on its optical center and has its own camera coordinate system as horizontal, vertical and optical axes. As shown in Figure 1, there is a point $M$ in the world coordinate system, and it is projected to multiple image points $m_{i j}$ respectively. According to the pinhole imaging principle, the relation of between $m_{i j}$ and $M$ is represented by the camera projection matrix, ${ }^{[26]}$ as equation (1).

$$
m_{i j}=p_{i j} M=K_{i j}\left[R_{i j} \mid T_{i j}\right] M
$$

$i$ represents the row index in the matrix camera arrangement, $j$ represents the column index in the matrix camera arrangement, $K, R, T$ represent the camera's intrinsic matrix, rotation matrix and translation matrix respectively, the coordinates of subpoint $m_{i j}$ is presented by $m_{i j}$ in the pixel coordinate system of image.

\subsection{Multi-view image rectification algorithm for matrix camera arrangement}

Image rectification is to calculate the new coordinates of each pixel in the rectified image. Point $m$ denotes one arbitrary point in original image and $m^{\prime}$ is the corresponding 
point in the rectified image. Their relationship can be expressed as

$$
m^{\prime}=H m
$$

Where $H$ is the homography matrix between the original image and the rectified image. ${ }^{[16]}$ This work focuses on calculating the homography matrix $H$. In this paper we define $H$ as the image rectifying transform matrix (IRTM).

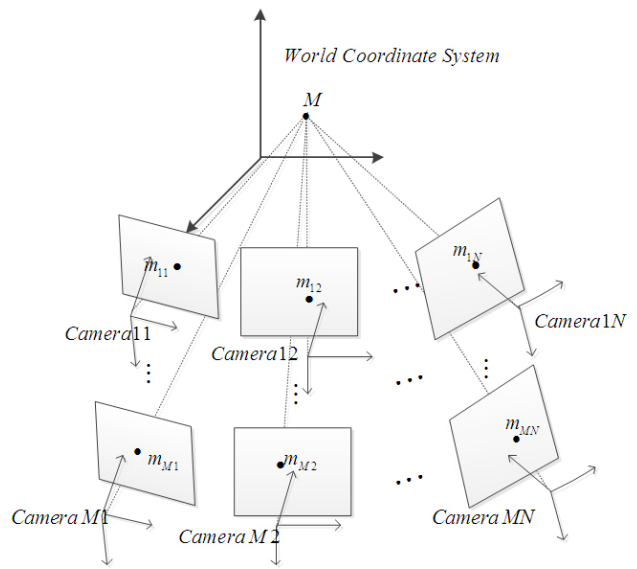

Figure 1: The projective model of matrix multi-camera arrangement

\subsubsection{Rectifying procedure}

The flow chart of the rectifying procedure of the proposed algorithm is shown in Figure 2. Firstly, we use the traditional Zhang's camera calibration algorithm ${ }^{[27]}$ to obtain intrinsic characteristics of cameras, the rotation matrixes and translation matrixes corresponding to the reference camera, respectively. Then, according to the actual camera parameters, we calculate the ideal intrinsic characteristics, rotation matrix and translation matrixes. Finally, we calculate rectifying transform matrix to rectify images based on actual parameters and ideal parameters. In Figure $2, K_{i j}$ represents the actual intrinsic matrix of the cameraij (the camera in the $\mathrm{i}-t h$ row and the $\mathrm{j}$-th column), $o m_{i j}$ and $T_{i j}$ are rotation matrix and translation matrix of relative to the reference camera camera11, respectively. $K_{\text {ideal }}, \mathrm{om}_{\text {ideal }}$ and $T_{i j-i d e a l}$ are the ideal intrinsic matrix, rotation matrix and translation matrix of cameraij, respectively.

\subsubsection{Calculation of ideal parameters for matrix multi- camera arrangement}

In order to solve the multi-view image rectifying transform matrix, we calculate ideal camera projection matrix by determining the following ideal parameters of cameras: ideal rotation matrix, ideal optical centers and ideal intrinsic characteristics of matrix multi-camera arrangement.

\section{Calculation of ideal rotation matrix}

There is a matrix multi-camera arrangement with $M$ rows and $N$ columns. We adopt the camera coordinate system $o-x_{11} y_{11} z_{11}\left(o x_{11}, o y_{11}\right.$ and $o z_{11}$ respectively represent horizontal axes, vertical axes, and optical axes of camera 11 located in first row and first column) as the reference coordinate system as shown in Figure 3. Then we get rotation matrix $o m_{i j}=\left(\overrightarrow{x_{i j}}, \overrightarrow{y_{i j}}, \overrightarrow{i_{i j}}\right)$ and translation matrix $T_{i j}$ of cameraij $(i=1,2 \ldots M ; j=1,2 \ldots N)$ with respect to camera11, as well as the camera's intrinsic matrix $K_{i j}$ by Zhang's camera calibration algorithm.

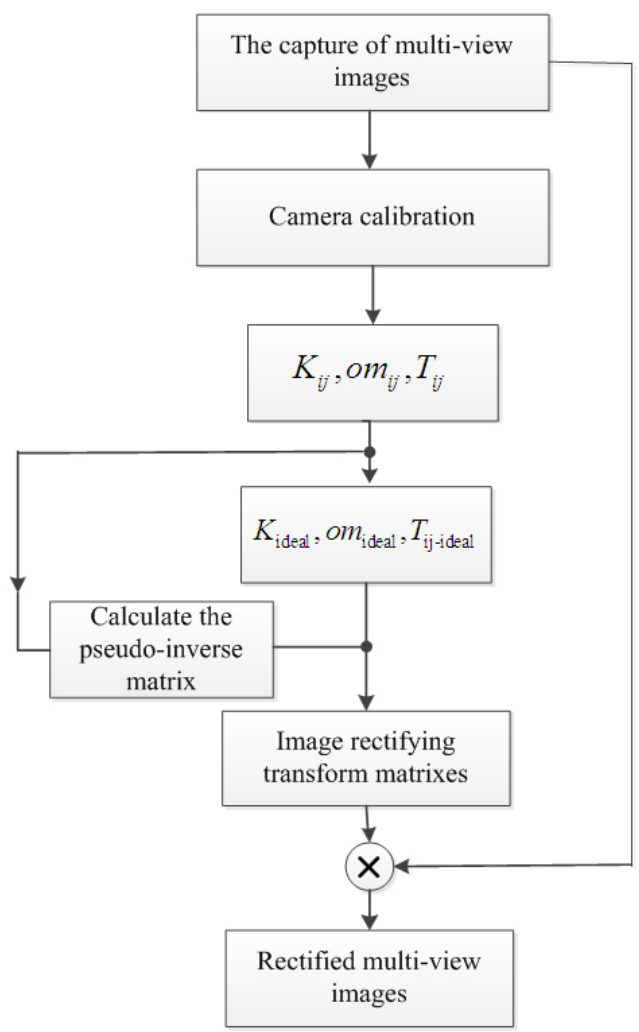

Figure 2: The flow chart of the image rectification procedure for matrix multi-camera arrangement

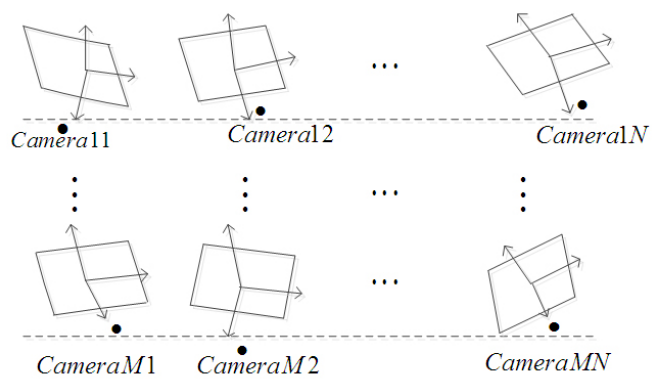

Figure 3: The actual matrix multi-camera arrangement

The ideal rotation matrix $o m_{\text {ideal }}$ needs to be firstly calculated. We adopt ergodic method to calculate ideal rotation matrix, which can improve the accuracy of rectification. In 
the procedure, $a$ is set to be any value in the range from $\min \left(x_{11} \ldots \overrightarrow{x_{i j} \ldots x_{M N}}\right)$ to $\max \left(\overrightarrow{x_{11} \ldots} \overrightarrow{x_{i j} \ldots x_{\vec{M} N}}\right)$, also, $b$ is set to be any value in the range from $\min \left(\overrightarrow{y_{11}} \ldots \overrightarrow{y_{i j}} \ldots y_{M N}\right)$ to $\max \left(\overrightarrow{y_{11}} \ldots \overrightarrow{y_{i j}} \ldots y_{\vec{M} N}\right)$, and $c$ is set to be any value in the range from $\min \left(\overrightarrow{z_{11}} \ldots \overrightarrow{z_{i j}} \ldots z_{M N}\right)$ to $\max \left(z_{11} \ldots \overrightarrow{z_{i j}} \ldots z_{\overrightarrow{M N}}\right)$. Then we can use equation (3) to solve virtual rotation matrix, which is synthetically the closest to all of actual cameras' rotation matrixes ,to get ideal rotation matrix.

$$
o m_{\text {ideal }}=\left[x_{i \overrightarrow{d e a l}}, y_{\text {ideal }}, z_{\text {ideal }}\right]=\arg \min \sum_{i=1}^{M} \sum_{j=1}^{N} \sqrt{\left(\vec{x}-\overrightarrow{x_{i j}}\right)^{2}+\left(\vec{y}-\overrightarrow{y_{i j}}\right)^{2}+\left(\vec{z}-\overrightarrow{z_{i j}}\right)^{2}} \quad(\vec{x} \in a, \vec{y} \in b, \vec{z} \in c)
$$

Selection of ideal optical centers of matrix multi-camera arrangement

On the basis of the obtained ideal rotation matrix, the next step is to calculate the ideal location of optical centers of matrix multi-camera arrangement, which stratifies the condition that optical centers are collinear in every row and every column. Besides all of the distance between adjacent optical centers in each row are $\vec{l}\left(\vec{l}=l x_{i \text { deal }}\right)$ and all of the distance between adjacent optical centers in each column are $\vec{d}\left(\vec{d}=d y_{i d e a l}\right) . C_{i j}(i=1,2 \ldots M ; j=1,2 \ldots N)$, as shown in Figure 4, is the actual position of optical centers of camera 11 in the $i-t h$ row and $j-t h$ column. We de- fine $C_{i j-i d e a l}$ as the ideal optical center of cameraij. If we select the first camera optical center of camera11 as reference, as shown in Figure 4, we can use equation (4) to solve $C_{i j}^{\prime}$. The coordinate of $C_{i j}^{\prime}$ is $C_{i j}^{\prime}=\left(x_{i j}^{\prime}, y_{i j}^{\prime}, z_{i j}^{\prime}\right)$ $(i=1,2 \ldots M ; j=1,2 \ldots N) . a^{\prime}$ is set to any value in the range from $\min \left(x_{11}^{\prime} \ldots x_{i j}^{\prime} \ldots x_{M N}^{\prime}\right)$ to $\max \left(x_{11}^{\prime} \ldots x_{i j}^{\prime} \ldots x_{M N}^{\prime}\right)$, also, $b^{\prime}$ is set to any value in the range from $\min \left(y_{11}^{\prime} \ldots y_{i j}^{\prime} \ldots y_{M N}^{\prime}\right)$ to $\max \left(y_{11}^{\prime} \ldots y_{i j}^{\prime} \ldots y_{M N}^{\prime}\right)$, and $c^{\prime}$ is set to any value in the range from $\min \left(z_{11}^{\prime} \ldots z_{i j}^{\prime} \ldots z_{M N}^{\prime}\right)$ to $\max \left(z_{11}^{\prime} \ldots z_{i j}^{\prime} \ldots z_{M N}^{\prime}\right)$. Firstly, we calculate the ideal optical center position of camera 11 according to formula (5), then, we get other cameras' ideal optical center position according to formula (6).

$$
\begin{aligned}
& C_{i j}^{\prime}=C_{i j}-(i-1) \vec{d}-(j-1) \vec{l} \quad(i=1,2 \ldots M, j=1,2, \ldots N) \\
& C_{11-i d e a l}=\arg \min \sum_{i=1}^{M} \sum_{j=1}^{N} \sqrt{\left(x-x_{i j}^{\prime}\right)^{2}+\left(y-y_{i j}^{\prime}\right)^{2}+\left(z-z_{i j}^{\prime}\right)^{2}} \quad\left(x \in a^{\prime}, y \in b^{\prime}, z \in c^{\prime}\right) \\
& C_{i j-\text { ideal }}=C_{11-\text { ideal }}+(i-1) \vec{d}+(j-1) \vec{l} \quad(i=1,2,3 \ldots M, j=1,2,3 \ldots N)
\end{aligned}
$$

Thus, we have the ideal camera centers, which not only satisfy the ideal condition but also minimize the sum of distances from the original camera centers. Using these camera centers, the translation vectors for the camera in the $i-t h$ row and $j-t h$ column of the matrix multi-camera arrangement can be defined as

$$
T_{i j-i d e a l}=-R_{i d e a l} C_{i j-i d e a l}
$$

where $R_{\text {ideal }}$ is Rodrigues transformation of $\mathrm{om}_{\text {ideal }}$.

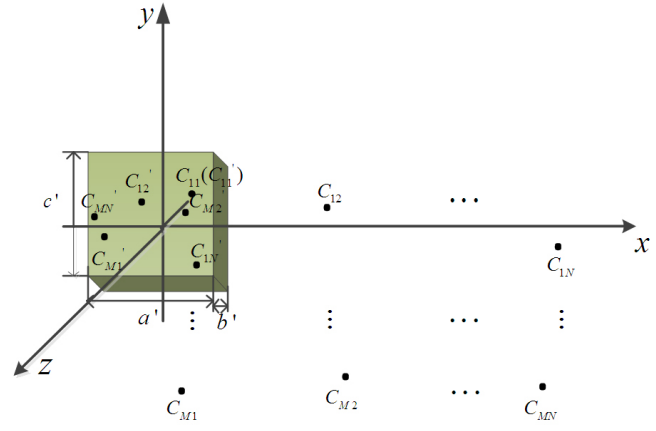

Figure 4: The cameras' optical centers diagram for actual matrix multi-camera arrangement

$$
\begin{array}{r}
f_{\text {ideal }}=\left[f_{\text {ideal }, x}, f_{\text {ideal }, y}\right]=\text { ave } \sum\left(f_{i j}\right)=\text { ave } \sum\left(\left[f_{i j, x}, f_{i j, y}\right]\right) \\
f_{i j}=\left[f_{i j, x}, f_{i j, y}\right] \in f_{i j}=\left[f_{i j, x}, f_{i j, y}\right] \mid\left(f_{i j}-\bar{f}\right)=\left(\left[f_{i j, x}, f_{i j, y}\right]-\left[\bar{f}_{x}, \bar{f}_{y}\right]\right) .^{2} \leq 2 \sigma^{\prime}
\end{array}
$$


Calculation of Common Intrinsic Matrix $K_{\text {ideal }}$ for Matrix Multi-camera Arrangement

The next term to be calculate is the ideal intrinsic matrix $K_{\text {ideal }}$. We mainly estimate the ideal focal length $f_{\text {ideal }}=\left[f_{\text {ideal }, x}, f_{\text {ideal }, y}\right]$ and the ideal principal point $P_{\text {ideal }}=\left[P_{\text {ideal }, x}, P_{\text {ideal }, y}\right]$. Firstly, we calculate the average $\bar{f}=\left(\bar{f}_{x}, \bar{f}_{y}\right)$ and the standard deviation $\sigma$ of actual focal lengths, and then exclude the focal length which is outside the region bounded by $\sqrt{2} \sigma$ based on $\bar{f}$. Finally we calculate the ideal focal length $f_{\text {ideal }}$ which is the average of the survived focal lengths.

The method of calculating principal point is the same as focal length. As shown in Equation (9), $\sigma^{\prime}$ denotes the standard deviation of the actual principal point coordinates.

$$
\begin{array}{r}
P_{\text {ideal }}=\left[P_{\text {ideal }, x}, P_{\text {ideal }, y}\right]=\text { ave } \sum\left(P_{i j}\right)=\text { ave } \sum\left(\left[P_{i j, x}, P_{i j, y}\right]\right) \\
P_{i j}=\left[P_{i j, x}, P_{i j, y}\right] \in P_{i j}=\left[P_{i j, x}, P_{i j, y}\right] \mid\left(P_{i j}-\bar{P}\right)=\left(\left[P_{i j, x}, P_{i j, y}\right]-\left[\bar{P}_{x}, \bar{P}_{y}\right]\right) .^{2} \leq 2 \sigma^{\prime}
\end{array}
$$

We then obtain the common intrinsic matrix $K_{\text {ideal }}$ from Equation (10). Since the skew parameter $S$ is almost to be zero, it has little effect on the image rectification, here we set it to be zero.

$$
K_{\text {ideal }}=\left[\begin{array}{ccc}
f_{\text {ideal }, x} & S & P_{\text {ideal }, x} \\
0 & f_{\text {ideal }, y} & \\
0 & 0 & 1
\end{array}\right]
$$

Using Equation (3), (7), (10), we finally obtain the camera's projection matrix of the ideal multi-camera arrangement as Equation (11). The multi-camera arrangement represented by Equation (11) satisfies the ideal geometric conditions, as shown in Figure 5.

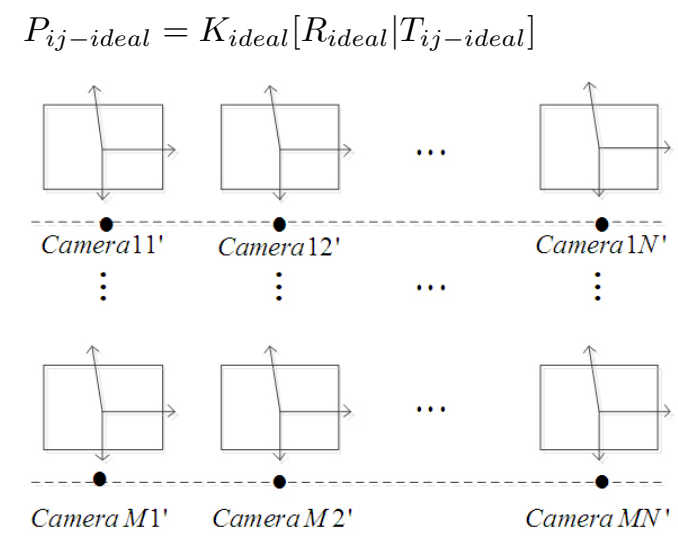

Figure 5: The schematic of ideal matrix multi-camera arrangement

\subsubsection{Calculation of the image rectifying transform ma- trix (IRTM)}

Since the epipolar geometry exists in each viewpoint, there is a point-to-point mapping called 2D homography $H$ from original image plane to ideal image plane. ${ }^{[26]}$ We employ this homography as the IRTM $H_{i j}$ for cameraij as Equation (12), where $P^{\dagger}$ is the pseudo inverse matrix of $P_{i j}$.

$$
H_{i j}=P_{i j-i d e a l} P_{i j}^{\dagger}
$$

\section{Experiments and data analysis}

In order to test the rectification effect of the proposed algorithm in this paper, we took multiple groups of images in experiment. We take the rectification effect of the following two groups of images as example for discussion. In this paper, we set up the matrix multi-camera arrangement with four rows and four columns. We captured two sets of images with resolution of $800 * 600$. The first group of images is taken by the matrix multi-camera arrangement whose distance between columns is $65 \mathrm{~mm}$ and distance between rows is $80 \mathrm{~mm}$, and shot the red kettle whose distance is about $2.5 \mathrm{~m}$ in front of the multi-camera array. The second group of images is captured by the matrix multi-camera arrangement whose distance between columns is $90 \mathrm{~mm}$ and distance between rows is $80 \mathrm{~mm}$, and shot the red kettle which distance is about $3 \mathrm{~m}$ in front of the multi-camera array.

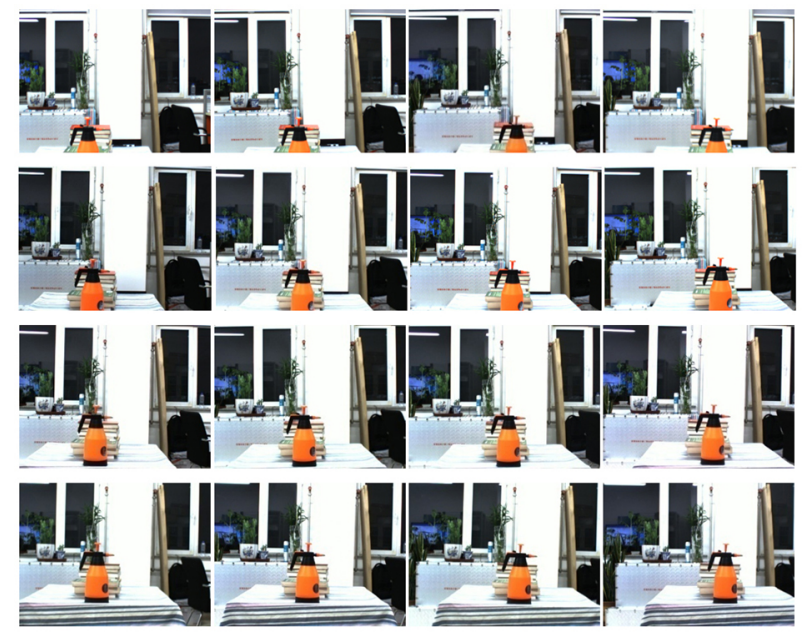

Figure 6: The first group of original images

\subsection{The rectification effect's analysis for the first group of images}

Figure 6 shows the first group of red kettle images captured by the matrix multi-camera arrangement with four rows and four columns whose distance between columns is $65 \mathrm{~mm}$ and 
distance between rows is $80 \mathrm{~mm}$. Figure 7 are the synthetic images of the original images for each row, where the blue dots represent the matching feature points of images. It can be seen from Figure 7, there exist geometric errors. There are vertical parallax and non-uniform horizontal disparities between the neighboring views in each row. Figure 8 are the synthetic images of the original images for each column. There are, as shown in Figure 8, horizontal pixel mismatches and non-uniform vertical disparities between the neighboring views in each column.
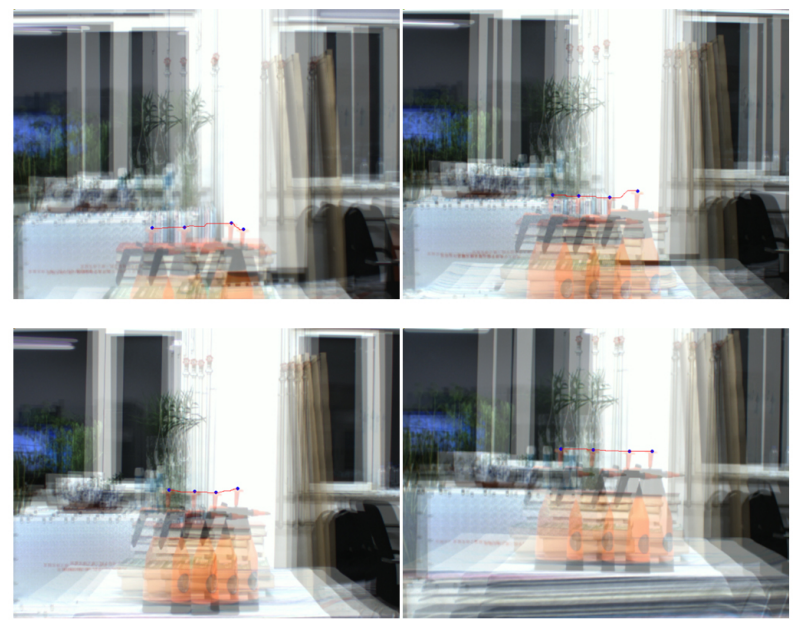

Figure 7: The synthetic images of the original images for each row(top left: the first row from the top; top right: the second row; low left: the third row; low right: the fourth row)
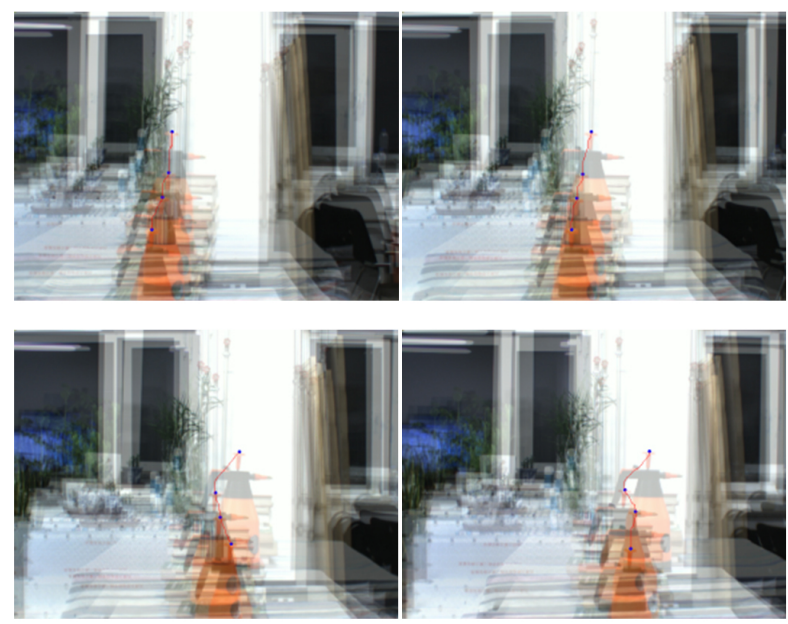

Figure 8: The synthetic images of the original images for each column(top left: the first column from the left; top right: the second column; low left: the third column; low right: the fourth column)

Figure 9 shows the first group of rectified images. Figure 10 are synthetic images of the rectified images for each row. It can be observed from Figure 10, the vertical parallax and the otherness of non-uniform horizontal disparity of the images in each row are remarkably reduced after the rectification with our algorithm. Figure 11 are the synthetic images of rectified images for each column. We observe that the horizontal parallax and the otherness of non-uniform vertical disparity of the images in each column are obviously reduced after the rectification.

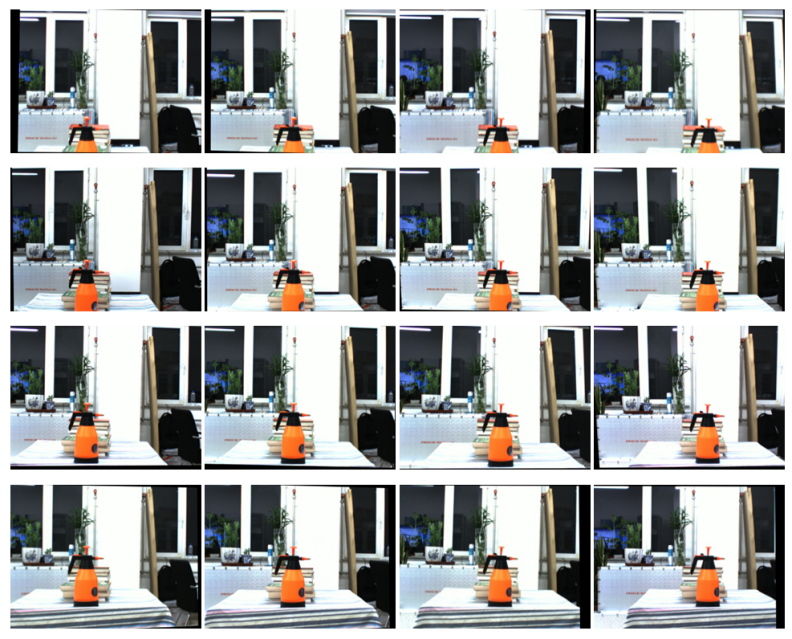

Figure 9: The first group of rectified images
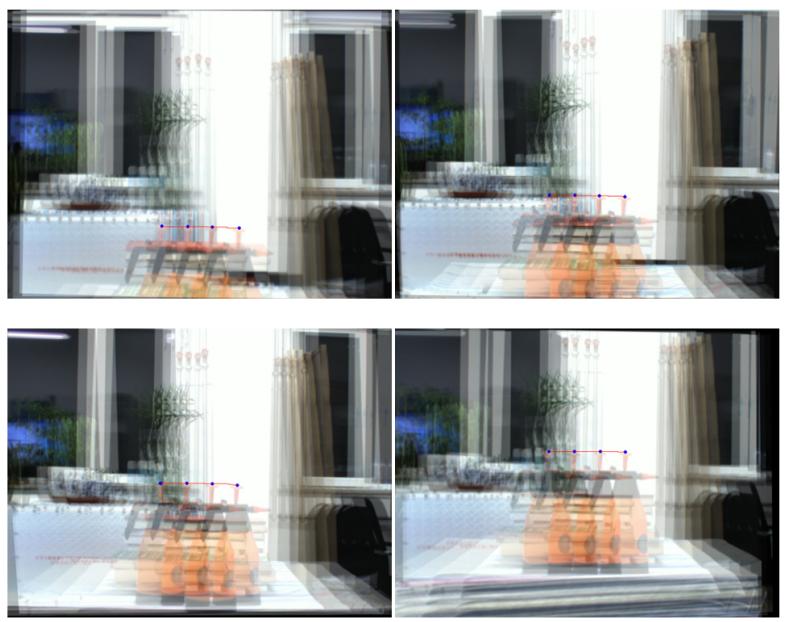

Figure 10: The synthetic images of the rectified images for each row

\subsection{Data analysis of rectification effect for the first group of images}

In order to calculate the vertical parallax of images in row and the horizontal parallax of images in column, we select one image in each row and in each column as reference image respectively, and then we adopt the SURF algorithm to detect and match corresponding points between reference image and other images in each row and in each column, respectively. ${ }^{[28]}$ We choose 30 corresponding points in advance. The outliers are detected using RANSAC applied 
on the epipolar geometry between each consecutive pair of images. We take the average of the absolute value of the Dvalue of survived corresponding points' vertical pixels as the vertical parallax between each image and reference image for the images in row, as shown in Figure 12(a).Similarly, we take the average of the absolute value of the D-value of survived corresponding points' horizontal pixels as the horizontal parallax between each image and reference image for the images in column, as shown in Figure 12(b). The method to calculate the horizontal parallax of the images in row and the vertical parallax of images in column is roughly the same as above. We adopt the SURF algorithm to detect and match corresponding points between each consecutive pair of images in each row and each column. We calculate the D-value of corresponding points' horizontal pixels as the horizontal parallax of adjacent images for the images in row. Similarly, we calculate the D-value of corresponding points' vertical pixels as the vertical parallax of adjacent images for the images in column.
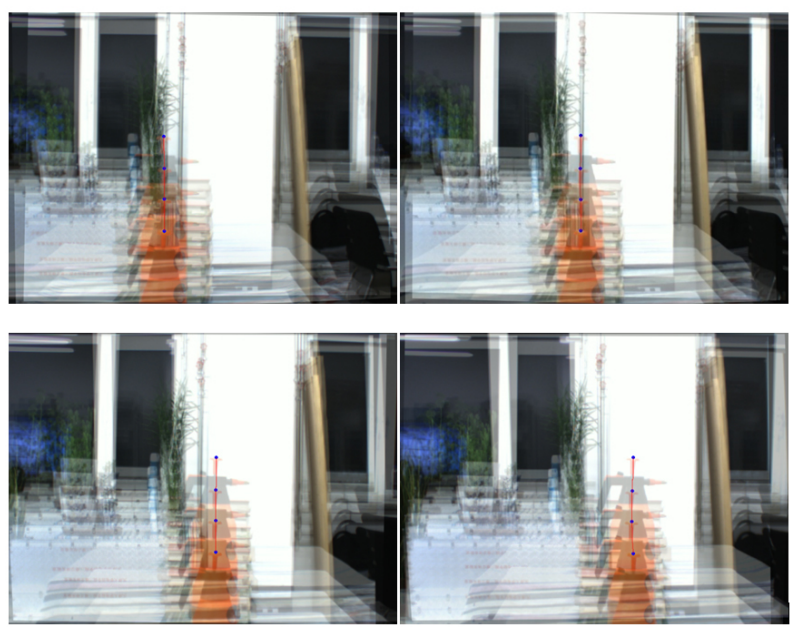

Figure 11: The synthetic images of the rectified images for each column

Table 1: The vertical parallax comparison for each row images.

\begin{tabular}{llllll}
\hline & $\begin{array}{l}\text { First } \\
\text { row }\end{array}$ & $\begin{array}{l}\text { Second } \\
\text { row }\end{array}$ & $\begin{array}{l}\text { Third } \\
\text { row }\end{array}$ & $\begin{array}{l}\text { Forth } \\
\text { row }\end{array}$ & Average \\
\hline Original (pixels) & 6.27 & 7.87 & 6.17 & 3.73 & 6.01 \\
Rectified(pixels) & 1.31 & 0.72 & 0.98 & 0.53 & 0.89 \\
\hline
\end{tabular}

Figure 13 gives the vertical parallax comparison between the original and rectified images for each row. We consider the leftmost end viewpoint as the reference point for each row. And we select the images captured by camera1, camera5,camera9, camera 13 as the reference images for each row, respectively. In Figure 13, the blue bar represents original vertical parallax between reference image and other images in each row, and the brown bar represents vertical parallax of rectified images. Then, we set the average value of the blue bars as original vertical parallax of images in each row, respectively. Similarly, we set the average value of the brown bars as vertical parallax of rectified images in each row, respectively. Vertical parallax comparison between original and rectified images is listed in Table 1.

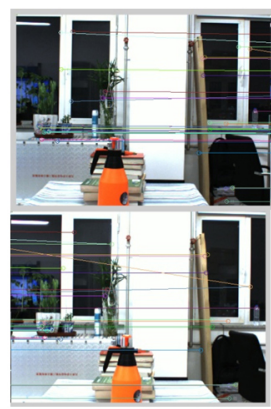

(a)

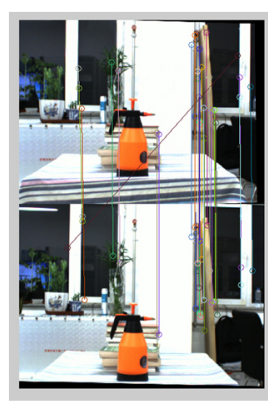

(b)
Figure 12: Detection and matching of corresponding feature points

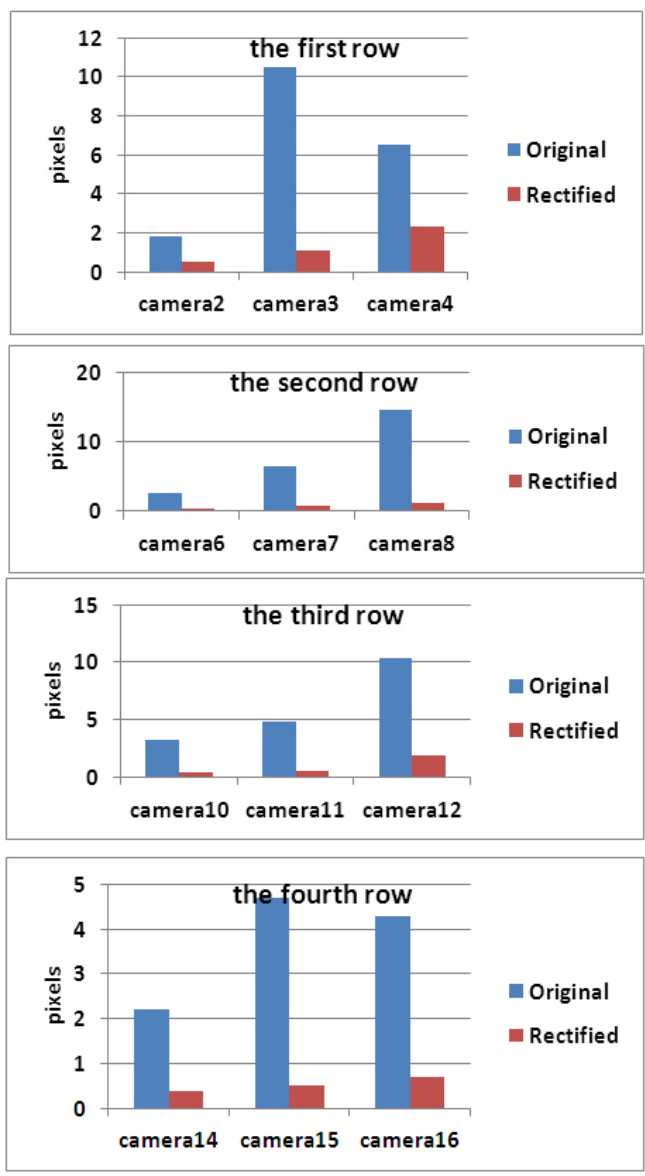

Figure 13: Vertical parallax comparison between original and rectified images for each row

Analysis of vertical parallax were performed above, we noticed that the proposed method efficiently reduced the verti- 
cal mismatches of images in each row. Then it comes to analysis of otherness of non-uniform horizontal parallax. This paper takes the Standard Deviation of horizontal parallax between each consecutive pair of images as the parameter evaluating otherness of non-uniform horizontal parallax for each row. In Figure 14, the blue line indicates the horizontal parallaxes of original images, and the red line indicates horizontal parallaxes of rectified images. In Figure 14 ,abscissa axis represents the consecutive pair of neighboring images in each row and vertical axis represents the value of horizontal parallax (unit: pixels). Otherness comparison of non-uniform horizontal parallax between original and rectified images for each row is listed in Table 2. Before rectification, disparity variation was large for each row. However, the proposed method almost uniform disparities for images in each row, as shown in Figure 14 and Table 2.

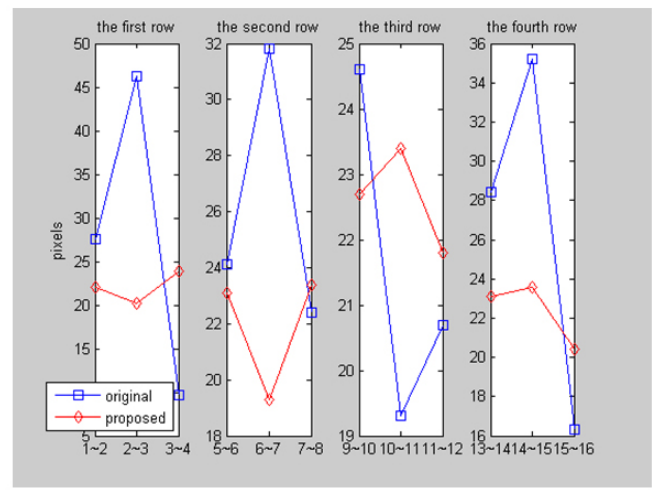

Figure 14: Horizontal parallax comparison between original and rectified images for each row

Table 2: Otherness comparison of non-uniform horizontal parallax between original and rectified images for each row

\begin{tabular}{llllll}
\hline & $\begin{array}{l}\text { First } \\
\text { row }\end{array}$ & $\begin{array}{l}\text { Second } \\
\text { row }\end{array}$ & $\begin{array}{l}\text { Third } \\
\text { row }\end{array}$ & $\begin{array}{l}\text { Forth } \\
\text { row }\end{array}$ & Average \\
\hline Original(pixels) & 14.9 & 4.08 & 2.42 & 7.68 & 7.72 \\
Rectified(pixels) & 1.51 & 1.87 & 0.65 & 1.50 & 1.38 \\
\hline
\end{tabular}

It is the analysis of rectification effect for each row images above, which shows that our algorithm effectively reduces the vertical parallax and otherness of non-uniform horizontal parallax. Then, we analyse the rectification effect of images in column. As the same with row images, we analyse the vertical parallax and horizontal parallax of original and rectified images for each column. In order to calculate the horizontal parallax, we consider the upmost viewpoint as reference point for each column. And we select the images captured by camera, camera 2 , camera 3 , camera 4 as reference images for each column, respectively. Figure 15 shows horizontal parallax comparison between original and rectified images for each column. In Figure 15, the blue bar represents original horizontal parallax between reference image and other images for each column, and the brown bar represents horizontal parallax of rectified images. The comparison of the average values of blue bars and brown bars is listed in Table 3. The horizontal parallax is effectively reduced after rectification for each column images, as shown in Table 3.
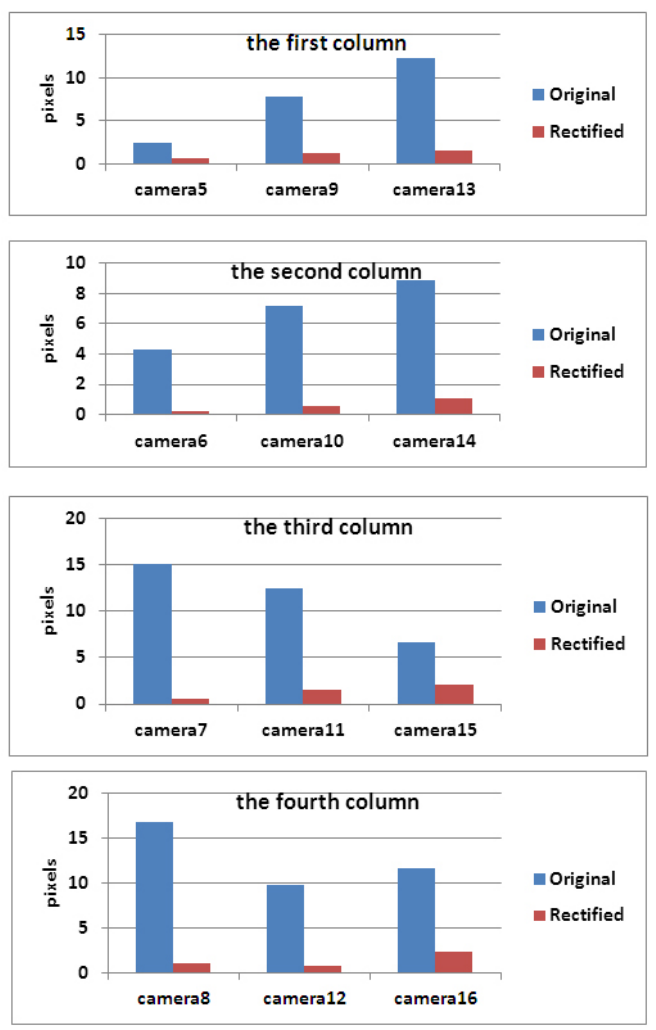

Figure 15: Horizontal parallax comparison between original and rectified images for each column

Table 3: Horizontal parallax comparison for each column images.

\begin{tabular}{llllll}
\hline & $\begin{array}{l}\text { First } \\
\text { column }\end{array}$ & $\begin{array}{l}\text { Second } \\
\text { column }\end{array}$ & $\begin{array}{l}\text { Third } \\
\text { column }\end{array}$ & $\begin{array}{l}\text { Forth } \\
\text { column }\end{array}$ & Average \\
\hline Original (pixels) & 7.50 & 6.82 & 11.39 & 12.77 & 9.62 \\
Rectified(pixels) & 1.13 & 0.63 & 1.40 & 1.37 & 1.13 \\
\hline
\end{tabular}

The analysis of horizontal parallax were performed above, we noticed that the proposed method efficiently reduced the horizontal mismatches of images in each column. Then it comes to the analysis of vertical parallax. Similarly, we take the Standard Deviation of vertical parallax between each consecutive pair of images as the parameter evaluating the otherness of non-uniform vertical parallax for each column. Figure 16 shows the vertical parallax comparison between original and rectified images for each column. The otherness comparison of non-uniform vertical parallax between original and rectified images for each column is listed in Table 4. It's obvious that the proposed method provides almost uniform disparity for each column images. 
Table 4: Otherness comparison of non-uniform vertical parallax between original and rectified images for each column.

\begin{tabular}{llllll}
\hline & $\begin{array}{l}\text { First } \\
\text { column }\end{array}$ & $\begin{array}{l}\text { Second } \\
\text { column }\end{array}$ & $\begin{array}{l}\text { Third } \\
\text { column }\end{array}$ & $\begin{array}{l}\text { Fourth } \\
\text { column }\end{array}$ & Average \\
\hline Original(pixels) & 6.90 & 8.51 & 10.01 & 7.94 & 8.34 \\
Rectified(pixels) & 1.10 & 0.79 & 0.83 & 1.25 & 0.99 \\
\hline
\end{tabular}

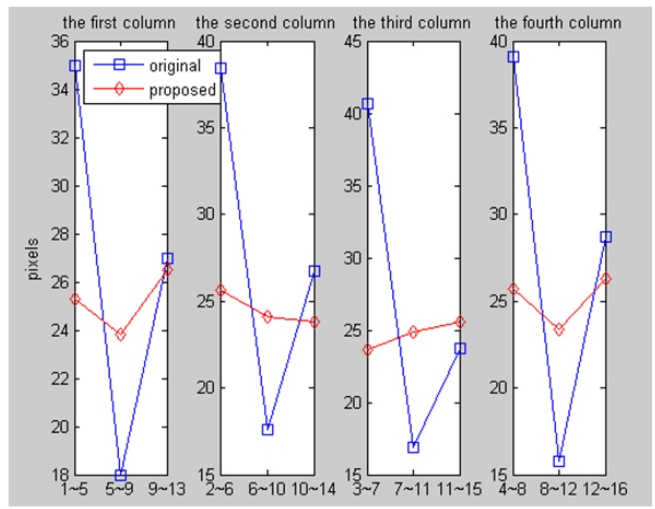

Figure 16: Vertical parallax comparison between original and rectified images for each column.

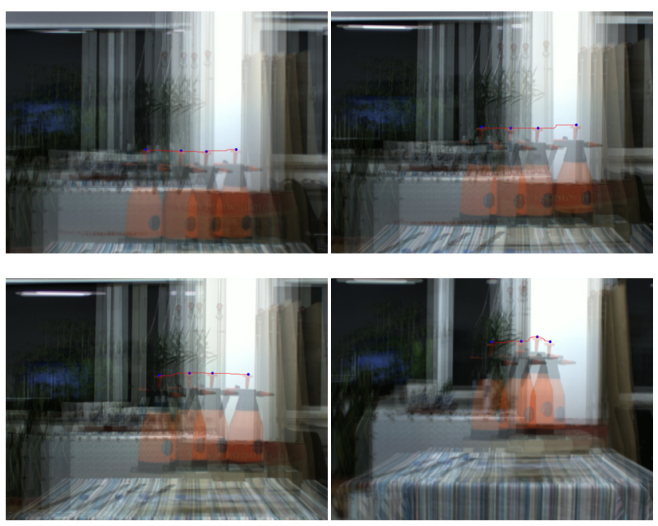

Figure 17: The synthetic images of the original images for each row.

\subsection{The Rectification Effect's Analysis for the Sec- ond Group of Images}

The second group of red kettle images is captured by the matrix multi-camera arrangement with four rows and four columns whose distance between columns is $90 \mathrm{~mm}$ and distance between rows is $80 \mathrm{~mm}$. Figure 17 are the synthetic images of the original images for each row. Figure 18 shows the synthetic images of the original images for each column. Figure 19 are the synthetic images of the rectified images for each row. And Figure 20 shows the synthetic images of the rectified images for each column. It can be seen from Figure 17 and Figure 19, before rectification, the vertical parallax and disparity variation of the original images in each row is large. However, the proposed method provides almost no vertical parallax and uniform disparity for row images. Similarly, the proposed algorithm provides almost no horizontal parallax and uniform disparity for column images. The effect of rectified images represents the characteristics of the ideal matrix multi-camera arrangement.
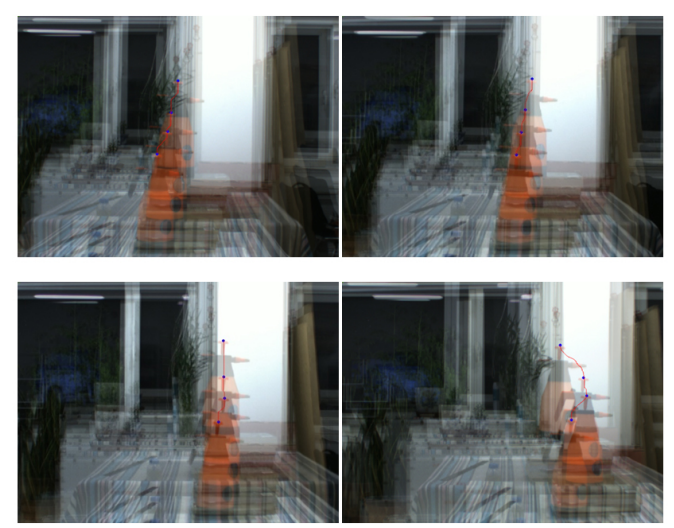

Figure 18: The synthetic images of the original images for each column.
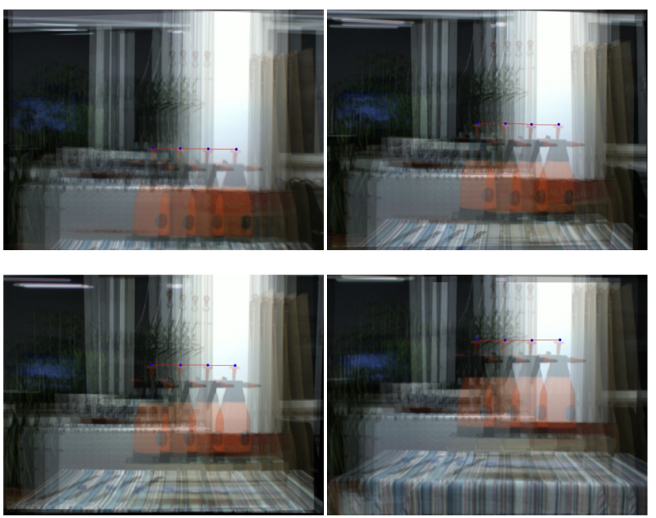

Figure 19: The synthetic images of the rectified images for each row.
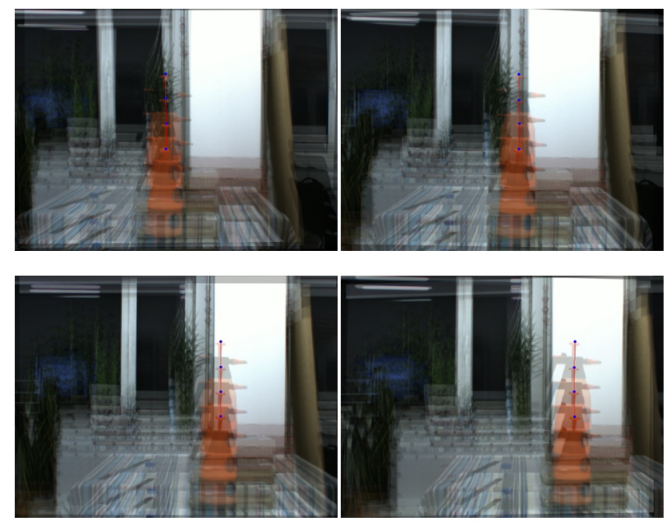

Figure 20: The synthetic images of the rectified images for each column. 


\subsection{Data analysis of the rectification effect for the second group of images}

Figure 21 gives the vertical parallax comparison between original and rectified images for each row. We select the images captured by camera 1 , camera5, camera 9 , camera 13 as reference images for each row, respectively. In Figure 21, the blue bar represents original vertical parallax between reference image and other images in each row, and the brown bar represents vertical parallax of rectified images. Vertical parallax comparison between original and rectified images is listed in Table 5.

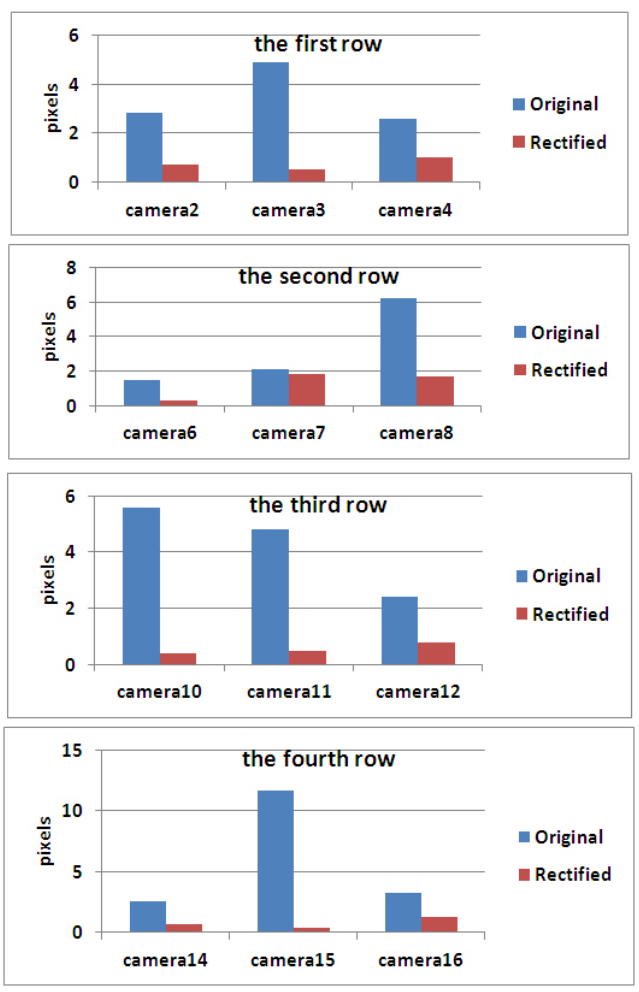

Figure 21: Vertical parallax comparison between original and rectified images for each row.

Table 5: Vertical parallax comparison between original and rectified images.

\begin{tabular}{llllll}
\hline & $\begin{array}{l}\text { First } \\
\text { row }\end{array}$ & $\begin{array}{l}\text { Second } \\
\text { row }\end{array}$ & $\begin{array}{l}\text { Third } \\
\text { row }\end{array}$ & $\begin{array}{l}\text { Forth } \\
\text { row }\end{array}$ & Average \\
\hline Original(pixels) & 3.43 & 3.27 & 4.27 & 5.83 & 4.20 \\
Rectified(pixels) & 0.73 & 1.30 & 0.57 & 0.81 & 0.85 \\
\hline
\end{tabular}

Figure 22 gives the horizontal parallax comparison between original and rectified images for each row. In Figure 22, the blue line indicates horizontal parallaxes between each consecutive pair of original images, and the red line indicates horizontal parallaxes between each consecutive pair of rectified images for each row. The otherness comparison of non-uniform horizontal parallax between original and rectified images for each row is listed in Table 6.

Published by Sciedu Press

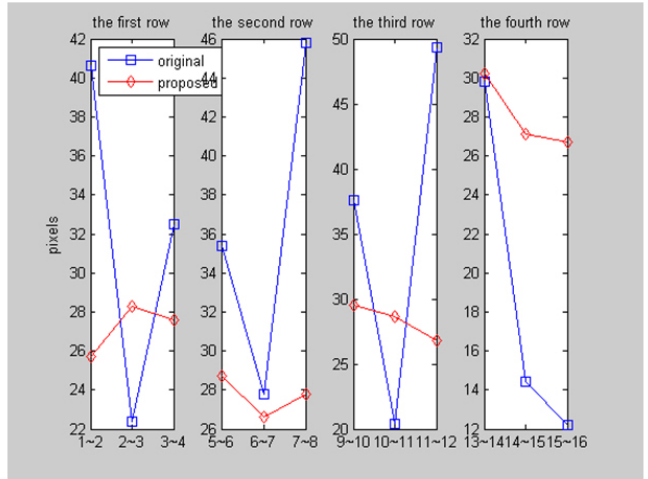

Figure 22: Horizontal parallax comparison between original and rectified images for each row.

Table 6: Otherness comparison of non-uniform horizontal parallax between original and rectified images for each row.

\begin{tabular}{llllll}
\hline & $\begin{array}{l}\text { First } \\
\text { row }\end{array}$ & $\begin{array}{l}\text { Second } \\
\text { row }\end{array}$ & $\begin{array}{l}\text { Third } \\
\text { row }\end{array}$ & $\begin{array}{l}\text { Fourth } \\
\text { row }\end{array}$ & Average \\
\hline Original(pixels) & 7.44 & 5.5 & 11.87 & 7.83 & 8.16 \\
Rectified(pixels) & 1.02 & 0.86 & 1.12 & 1.56 & 1.14 \\
\hline
\end{tabular}

Rectification effect analysis for each row images indicates that our algorithm effectively reduces vertical parallax and otherness of non-uniform horizontal parallax for row images. Then, we analyse the rectification effect for images in column. In order to calculate horizontal parallax, we consider the upmost viewpoint as reference point for each column. And we select the images captured by camera 1 , camera2, camera3, camera 4 as reference images for each column, respectively. Figure 23 shows horizontal parallax comparison between original and rectified images for each column. The horizontal parallax comparison for each column images is listed in Table 7. Figure 24 gives vertical parallax comparison between original and rectified images for each column. In Figure 24, the blue line indicates vertical parallaxes between each consecutive pair of original images, and the red line indicates vertical parallaxes between each consecutive pair of rectified images for each column. The otherness comparison of non-uniform vertical parallax between original and rectified images for each column is listed in Table 8.

Table 7: Horizontal parallax comparison for each column images.

\begin{tabular}{llllll}
\hline & $\begin{array}{l}\text { First } \\
\text { column }\end{array}$ & $\begin{array}{l}\text { Second } \\
\text { column }\end{array}$ & $\begin{array}{l}\text { Third } \\
\text { column }\end{array}$ & $\begin{array}{l}\text { Forth } \\
\text { column }\end{array}$ & Average \\
\hline Original(pixels) & 10.93 & 8.41 & 3.23 & 21.32 & 10.97 \\
Rectified(pixels) & 1.37 & 0.90 & 0.72 & 1.61 & 1.15 \\
\hline
\end{tabular}

The experimental results illustrate that our method effectively reduces vertical parallax and otherness of nonuniform horizontal parallax for row images, and markedly reduces horizontal parallax and otherness of non-uniform 
vertical parallax for column images.
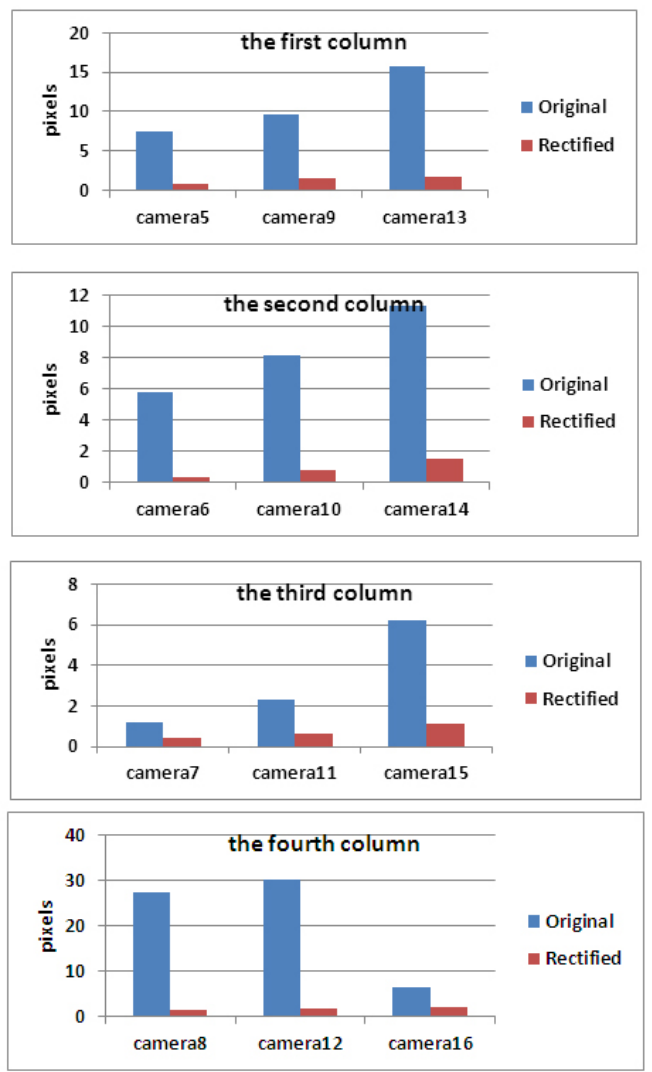

Figure 23: Horizontal parallax comparison between original and rectified images for each column.

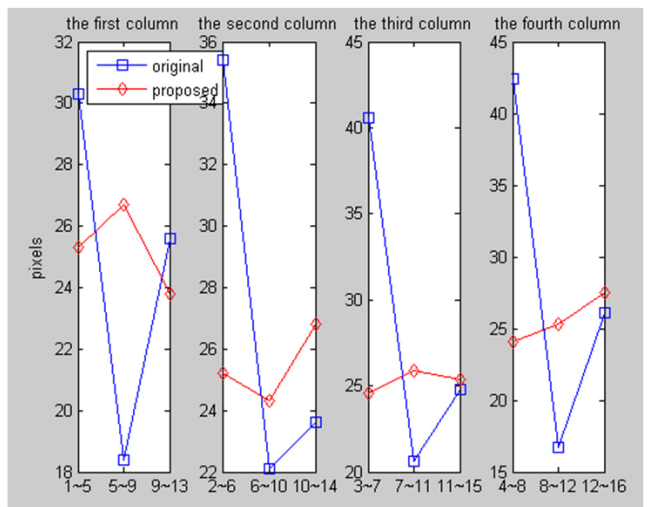

Figure 24: Vertical parallax comparison between original and rectified images for each column.

In order to test our algorithm sufficiently, we took multiple groups of images and obtain statistical result. Vertical parallax maintains within 1.5 pixels and otherness of horizontal parallax keeps within 2.0 pixels for row images rectified by our algorithm. And horizontal parallax maintains within 1.8 pixels and otherness of vertical parallax keeps within 2.0 pixels for column images. The statistical result shows that the proposed algorithm has strong robustness. Even if the matrix camera arrangement is in relatively large error position compared to the ideal condition, the rectification effect is still favorable. The quality of rectified images achieves the characteristics of the images captured by ideal matrix multi-camera arrangement.

Table 8: Otherness comparison of non-uniform vertical parallax between original and rectified images for each column.

\begin{tabular}{llllll}
\hline & $\begin{array}{l}\text { First } \\
\text { column }\end{array}$ & $\begin{array}{l}\text { Second } \\
\text { column }\end{array}$ & $\begin{array}{l}\text { Third } \\
\text { column }\end{array}$ & $\begin{array}{l}\text { Forth } \\
\text { column }\end{array}$ & Average \\
\hline Original(pixels) & 4.90 & 5.98 & 8.60 & 10.62 & 7.53 \\
Rectified(pixels) & 1.18 & 1.03 & 0.54 & 1.41 & 1.04 \\
\hline
\end{tabular}

In order to test the stability of numerical computation of our algorithm, each group of images was experiment for five times. The same corresponding points of each group of images were selected to experiment twice and the results indicated that acquired data of rectified images are the same. Moreover, we also selected other corresponding points for three times and the data are approximately the same. Our experiment results evidently illustrates that numerical computation of the proposed algorithm is highly stable.

We implemented our method in PC with a $3 \mathrm{GHZ}$ Intel Dual Core CPU and 3GB memory. After scene capturing and multi-camera calibration, the processing time to calculate the image rectifying transform matrix (IRTM) for views of 4 rows $\times 4$ columns is 0.196 seconds. The required time from applying the IRTM to the original image to obtaining the rectified image for one view is 0.022 seconds. This result shows that our method provides more than 45 frames per second of rectified images for each view.

\section{Conclusion}

This paper proposed an efficient and accurate multi-view image rectification algorithm for matrix multi-camera arrangement. Firstly, we used the ergodic method to obtain the ideal rotation matrix and the desired location of optical centers where the rectified images undergoes little deformation and have high accuracy. Then, we calculate the ideal projection matrix based on ideal rotation and ideal optical centers' location. Next, we calculate the rectifying transform matrix. Finally, using the rectifying transform matrix, we further rectify multi-view images. Moreover, there is an opportunity to select unexpected baseline that either minimizes the sum of absolute difference from each camera centers or is confirmed by a camera's horizontal axis in previous algorithm for parallel camera arrangement. Our algorithm for matrix camera arrangement overcomes the shortcoming of the previous algorithm for parallel camera arrangement, which guarantees that our algorithm has stronger robustness. By applying our algorithm to rectify original images, we efficiently reduced geometric errors and obtained rectified multi-view images without visual distortion, which 
achieve the characteristics of the images captured by the ideal matrix multi-camera arrangement.

\section{Acknowledgements}

This research is partially supported by the National Natural Science Foundation of China (No. 61101224),
Natural Science Foundation of Tianjin (No. 12jcqnjc00500), Supported by Program for New Century Excellent Talents in University(NCET-12-0400), Postdoctoral Fund in China(2012M520574) and China Aviation Fund(2012ZC48003).

\section{References}

[1] Tanimoto, M., Tehrani, M. P., Fujii, T., and Yendo, T. FTV for 3-D spatial communication. Proceedings of the IEEE, 2012; 100(4): 905-917. http://ieeexplore.ieee.org/xpls/abs_ all $\cdot$ jsp?arnumber $=6157594 \&$ tag $=1$

[2] Solh, M., and AlRegib, G. Hierarchical Hole-Filling For DepthBased View Synthesis in FTV and 3D Video. Selected Topics in Signal Processing, 2012; 6(5): 495-504. http: //ieeexplore.ieee. org/xpls/abs_all.jsp?arnumber $=6217270$

[3] Hubert, H., Stabernack, B., and Zilly, F. Architecture of a Low Latency Image Rectification Engine for Stereoscopic 3D HDTV Processing. Circuits and Systems for Video Technology, 2013; 23(5):813-822. http://ieeexplore.ieee.org/xpls/ abs_all.jsp?arnumber $=6328256$

[4] ISO/IEC JTC1/SC29/WG11 N5877. Applications and Requirements for 3DAV. 2003.

[5] ISO/IEC JTC1/SC29/WG11 N8944. Preliminary FTV model and requirements. 2007.

[6] ISO/IEC JTC1/SC29/WG11 M20068. New multi-view test sequences and camera parameters for $3 \mathrm{DV}$ standardisation work 2011.

[7] Barrera, C. F., Lumbreras, R. F., and Sappa, A. D. Multimodal Stereo Vision System: 3D Data Extraction and Algorithm Evaluation. Selected Topics in Signal Processing, 2012; 6(5): 437-446. http://ieeexplore.ieee.org/xpls/abs_ all.jsp?arnumber $=6220232$

[8] Koo, H. Segmentation and rectification of pictures in the cameracaptured images of printed documents.Multimedia, 2013; 15(3): 647 - 660. http://ieeexplore.ieee.org/xpls/abs_all. jsp?arnumber $=6392967$

[9] Liu, F., Niu, Y., and Jin, H. Casual Stereoscopic Photo Authoring. IEEE Transactions on Multimedia, 2013; 15(1): 129-140. http://ieeexplore.ieee.org/xpls/abs_all. jsp?arnumber $=6331540$

[10] Bangchang, P. N., Fujii, T., and Tanimoto, M. Experimental system of free viewpoint television. In Proc. of SPIEVol, 2003; 5006: 554-563. http://proceedings.spiedigitallibrary. org/proceeding . aspx?articleid=755653

[11] Fujii, T., and Tanimoto, M. A real-time ray-space acquisition system. In Electronic Imaging, 2004: 179-187. International Society for Optics and Photonics. 2004 http: //proceedings.spiedigitallibrary.org/ proceeding . aspx?articleid $=836687$

[12] Tanimoto, M., Tehrani, M. P., Fujii, T., and Yendo, T. Free-viewpoint TV. Signal Processing Magazine, IEEE, 2011; 28(1): 67-76. http://ieeexplore.ieee.org/xpl/freeabs_ all $\cdot$ jsp? arnumber $=5670636 \&$ reas on=concurrency

[13] Wilburn, B., Joshi, N., Vaish, V., Talvala, E. V., Antunez, E., Barth, A., ....and Levoy, M. High performance imaging using large camera arrays. In ACM Transactions on Graphics (TOG), 2005; 24(3): 765776 ACM. http://dl .acm.org/citation. cfm?id=1073259

[14] Bermudez-Cameo, J., Puig, L., and Guerrero, J. J. Hypercatadioptric line images for $3 \mathrm{D}$ orientation and image rectification. Robotics and Autonomous Systems, 2012; 60(6): 755-768. http://www.sciencedirect.com/science/article/pii/ S0921889012000449
[15] Loop, C., and Zhang, Z. Computing rectifying homographies for stereo vision.In Computer Vision and Pattern Recognition, 1999. (Vol. 1). IEEE. 1999. http://ieeexplore.ieee.org/xpls/ abs_all.jsp?arnumber $=786928$

[16] Fusiello, A., Trucco, E., and Verri, A. A compact algorithm for rectification of stereo pairs. Machine Vision and Applications, 2000; 12(1): 16-22. http://link.springer.com/article/ 10.1007/s001380050120

[17] Fusiello, A., and Irsara, L. Quasi-Euclidean epipolar rectification of uncalibrated images. Machine Vision and Applications, 2011; 22(4): 663-670. http://link.springer.com/article/ 10.1007/s00138-010-0270-3

[18] Koufogiannis, E. T., Sgouros, N. P., and Sangriotis, M. S. Robust integral image rectification framework using perspective transformation supported by statistical line segment clustering. Applied Optics, 2011; 50(34): H265-H277. http://www.opticsinfobase.org/ ao/fulltext . cfm?uri=ao-50-34-H265\&id=225644

[19] Banno, A., and Ikeuchi, K. Estimation of F-Matrix and image rectification by double quaternion. Information Sciences, 2012; 183(1): 140-150. http://www.sciencedirect.com/science/ article/pii/S0020025511004324

[20] Sun, C. Uncalibrated three-view image rectification. Image and Vision Computing, 2003; 21(3): 259-269. http://www.sciencedirect.com/science/article/ pii/S0262885602001579

[21] Wang, D., Lim, K. B., and Kee, W. L. Geometrical approach for rectification of single-lens stereovision system with a triprism. Machine Vision and Applications, 2013; 24(4): 1-13. http://link. springer.com/article/10.1007/s00138-012-0467-8

[22] Kim, D. , Fukushima, N., Yendo, T. , Tehrani, M.P. , Fujii, T., and Tanimoto, M. Rectification of camera array using bundle adjustment. in Proc. International Workshop on Advanced Image Technology, 2011: 1-6.

[23] Boutarel, F., and Nozick, V. Epipolar rectification for autostereoscopic camera setup. EAM Mechatronics. 2010: 133136. http://hal .archives-ouvertes.fr/docs/00/73/33/ 74/PDF/boutarel_EAM_2010.pdf

[24] Kang, Y. S., and Ho, Y. S. Geometrical compensation for multi-view video in multiple camera array. In ELMAR, 2008. 50th International Symposium (Vol. 1, pp. 83-86). IEEE. 2008. http://ieeexplore.ieee.org/xpls/abs_all. jsp?arnumber $=4747443$

[25] Kang, Y. S., and Ho, Y. S. An efficient image rectification method for parallel multi-camera arrangement. Consumer Electronics, 2011; 57(3): 1041-1048. http://ieeexplore.ieee.org/xpls/abs_ all. jsp?arnumber $=6018853$

[26] Hartley, R., and Zisserman, A. Multiple view geometry in computer vision (Vol. 2). Cambridge University Press. 2000.

[27] Zhang, Z. A flexible new technique for camera calibration. Pattern Analysis and Machine Intelligence, 2000; 22(11): 13301334. http://ieeexplore.ieee.org/stamp/stamp.jsp?tp= \&arnumber $=888718$

[28] Evans, C. Notes on the opensurf library. University of Bristol, Tech. Rep. CSTR-09-001. 2009. http://www. chrisevansdev . com/computer-vision-opensurf .html 